\title{
Command-Filtered Backstepping Control for a Class of Chaotic Systems
}

\author{
Chunxiang Sun \\ Department of Finance and Mathematics, Huainan Normal University, Huainan 232038, China \\ Correspondence should be addressed to Chunxiang Sun; sunchunxiang2000@163.com
}

Received 15 November 2021; Accepted 4 January 2022; Published 17 January 2022

Academic Editor: Ahmed Mostafa Khalil

Copyright (c) 2022 Chunxiang Sun. This is an open access article distributed under the Creative Commons Attribution License, which permits unrestricted use, distribution, and reproduction in any medium, provided the original work is properly cited.

The problem of command-filtered backstepping control for a class of chaotic systems was studied in this paper. At each step of the backstepping design, a command filtering backstepping method is introduced to use a new virtual control signal, in which the derivative of the virtual control was approximated by using the output of filter. Then, the asymptotic stability conditions for synchronization errors are obtained by using the Lyapunov stability criterion and this control method. The design technique has the advantages of the conventional command-filtered backstepping control. An example shows the proposed method was feasible.

\section{Introduction}

Chaos phenomenon is a seemingly irregular and inherently random complex phenomenon in a system, which widely exists in meteorology, water culture, communications, economy, and other fields. In recent years, chaotic systems have been studied deeply, especially in the field of chaos control. Chaos control mainly includes two purposes: to suppress chaos when it is not needed in practical application and to enhance chaos when it is needed. The idea of effectively controlling chaos is to control the chaotic phenomenon in the system to an ideal state by using small changes so that the chaotic phenomenon can be used effectively. Controlling chaos is the premise of making effective use of chaos. Therefore, controlling chaotic system to achieve the expected state has become one of the research focuses of scholars [1-5].

Since the development contribution of PC in synchronizing chaotic systems, synchronizing chaotic systems has aroused great interest of people, such as adaptive feedback control, backstepping control, impulse control, sliding mode control, fuzzy control, and other methods, which have been proposed [6-12]. In [13], the trajectory tracking control problem of mobile robot is studied. The trajectory tracking problem is transformed into the stabilization problem of error system through coordinate transformation. Based on the idea of feedback linearization and variable structure control, a composite finite time variable structure linearization tracking control is proposed. Backstepping control is one of the most common methods to deal with chaotic systems [14]. Therefore, this paper mainly uses the same method to eliminate the nonlinear term in the error to study the stability and asymptotic synchronization of chaotic systems.

It is important to note as well that the backstepping control method of chaotic systems has caught scholars' much attentions in recent years $[15,16]$. The control of pure feedback nonlinear systems is studied by using backstepping adaptive control and neural network adaptive control algorithms [17]. It is well known that for all kinds of control systems, there have been plenty of research achievements in different integer-order systems with the backstepping control method. Even so, there is a particular limitation of the traditional backstepping algorithm method. The limitation is that the backstepping design procedure has the problem of "explosion of complexity" [18-20]. This means that the derivative of the virtual control function increases with the increase of the system order, which makes the calculation prohibitive [21, 22]. Based on the backstepping control method, Li and Tong [23] studied the output regulation of strict feedback uncertain control system and finally obtained the global asymptotic stability. 
In this paper, we will study the finite time tracking control problem of command-filtered backstepping method for nonlinear systems. When designing each step of the backstepping, the command filter is replaced by the filter with asymptotic convergence rate, which can ensure that the output of the filter is similar to the derivative of the virtual control. At the same time, choosing proper control parameters is a better method. Compared with the proposed virtual control signal, the finite time virtual control signal can make the closed-loop system have faster response speed and higher tracking accuracy in [21, 22]. Using a coordinate transformation and based on the idea of backstepping design, the stabilization problem of a class of nonlinear systems with triangular structure is studied. Through the designed virtual control law, the controller is obtained [24].

Many results on controller design have been used to study the stability of systems with nonlinear inputs. In [25], the adaptive fuzzy control problem of single input single output nonlinear stochastic systems in the form of nonstrict feedback was investigated, in which the fuzzy logic system is used to approximate the uncertain nonlinear function, and the adaptive fuzzy controller is constructed by backstepping technology. This shows that there is still a lot of room for research integer-order systems with nonlinear inputs in the future. In a word, the study of the stability of systems with nonlinear inputs has certain theoretical value. Sui et al. [26] studied the finite time control of uncertain MIMO nontriangular random nonlinear systems and proposed the switching control strategy by using the finite time backstepping algorithm and the general Lyapunov function method. In the simulation results, the integer-order gyro system is synchronized. In $[27,28]$, the quasisynchronization problems of delayed stochastic multiplex networks and coupled system of $\mathrm{N}$ heterogeneous harmonic oscillators are discussed, respectively.

Inspired by the above discussions, the finite time tracking control problem of command-filtered backstepping method for nonlinear systems. The main contributions can be summarized as follows: (1) a command filtering backstepping method is introduced to use a new virtual control signal, in which the derivative of the virtual control was approximated by using the output of filter. (2) Compared with the proposed virtual control signal, the finite time virtual control signal can make the closed-loop system have faster response speed and higher tracking accuracy.

\section{Problem Formulation and Preliminaries}

Suppose the master chaotic system as,

$$
\left\{\begin{array}{l}
\dot{x}_{1}(t)=x_{2}(t)+f_{1}\left(\widetilde{x}_{1}(t)\right) \\
\dot{x}_{2}(t)=x_{3}(t)+f_{2}\left(\widetilde{x}_{2}(t)\right) \\
\vdots \\
\dot{x}_{n-1}(t)=x_{n}(t)+f_{n-1}\left(\tilde{x}_{n-1}(t)\right) \\
\dot{x}_{n}(t)=f_{n}(x(t))
\end{array}\right.
$$

where $x(t)=\left[x_{1}(t), \ldots, x_{n}(t)\right]^{T} \in R^{n}$ is the measurable state variable vector, $\tilde{x}_{i}(t)=$ $\left[x_{1}(t), x_{2}(t), \ldots, x_{i}(t)\right]^{T} \in R^{i}, i \in(1, \ldots, n)$, and $\tilde{x}_{n}(t)=$ $x(t)$. The smooth nonlinear functions $f_{i}\left(\tilde{x}_{i}(t)\right) \in R$ are assumed to be known, $i \in(1, \ldots, n)$.

Let the slave system be,

$$
\left\{\begin{array}{l}
\dot{y}_{1}(t)=y_{2}(t)+g_{1}\left(\tilde{y}_{1}(t)\right), \\
\dot{y}_{2}(t)=y_{3}(t)+g_{2}\left(\tilde{y}_{2}(t)\right), \\
\vdots \\
\dot{y}_{n-1}(t)=y_{n}(t)+g_{n-1}\left(\tilde{y}_{n-1}(t)\right), \\
\dot{y}_{n}(t)=g_{n}(y(t))+u(t),
\end{array}\right.
$$

where $y(t)=\left[y_{1}(t), \ldots, y_{n}(t)\right]^{T} \in R^{n}$ is the measurable state variable vector, $\quad \widetilde{y}_{i}(t)=\left[y_{1}(t), \quad y_{2}(t), \ldots\right.$, $\left.y_{i}(t)\right]^{T} \in R^{i}, i \in(1, \ldots, n)$, and $\tilde{y}_{n}(t)=y(t)$. The smooth nonlinear functions $g_{i}\left(\tilde{y}_{i}(t)\right) \in R$ are assumed to be known, $i \in(1, \ldots, n) . u(t) \in R$ is the control input.

Let $e(t)=\left[y_{1}(t)-x_{1}(t), \ldots, y_{n}(t)-x_{n}(t)\right]^{T}$, then the error dynamics is as follows:

$$
\left\{\begin{array}{l}
\dot{e}_{1}(t)=e_{2}(t)+l_{1}\left(\widetilde{x}_{1}(t), \widetilde{y}_{1}(t)\right), \\
\dot{e}_{2}(t)=e_{3}(t)+l_{2}\left(\widetilde{x}_{2}(t), \widetilde{y}_{2}(t)\right), \\
\vdots \\
\dot{e}_{n-1}(t)=e_{n}(t)+l_{n-1}\left(\widetilde{x}_{n-1}(t), \tilde{y}_{n-1}(t)\right), \\
\dot{e}_{n}(t)=l_{n}(x(t), y(t))+u(t),
\end{array}\right.
$$

where $l_{i}\left(\tilde{x}_{i}(t), \tilde{y}_{i}(t)\right)=g_{i}\left(\tilde{y}_{i}(t)\right)-f_{i}\left(\tilde{x}_{i}(t)\right), i \in(1, \ldots, n)$ and $l_{n}\left(\tilde{x}_{n}(t), \tilde{y}_{n}(t)\right)=l_{n}(x(t), y(t))=g_{n}(y(t))-f_{n}(x(t))$. For convenience, let us replace $l_{i}\left(\tilde{x}_{i}(t), \tilde{y}_{i}(t)\right)$ with $l_{i}\left(\tilde{w}_{i}(t)\right)$, $i \in(1, \ldots, n-1)$ and $l_{n}\left(\widetilde{w_{n}}(t)\right)=l_{n}(\widetilde{w}(t))=l_{n}(x(t), y(t))$.

Remark 1 (see [29]). In reality, the strict feedback system is usually expressed in the form of compact function. For example, (1) and (2) are strict feedback systems. Therefore, system (3) is also a strict feedback system. There are a lot of chaotic systems that can be expressed in (3), such as Chen system, Chua's circuit, and Arneodo system. In fact, the chaotic systems mentioned above are not strict feedback forms. However, we can rewrite it into (3) form through simple variable transformation.

Definition 1 (see [30]). If $\varphi:[0, b) \longrightarrow \Xi$ is a strictly increasing continuous function and $\varphi(0)=0$, then $\varphi$ belongs to class- $\mathscr{K}$.

Lemma 1. Let

$$
\dot{\mu}(\tau)=\Phi(\tau, \mu(\tau)),
$$

where $\Phi(\tau, \mu(\tau))$ is a Lipschitz continuous nonlinear function and 0 is an equilibrium point of (4). If there exists a Lyapunov function $V(\tau, \mu(\tau))$, $\mathscr{K}$ functions $\varphi_{i}(i=1,2,3)$ such that

$$
\begin{aligned}
& \varphi_{1}(\|\mu(\tau)\|) \leq V(\tau, \mu(\tau)) \leq \varphi_{2}(\|\mu(\tau)\|), \\
& \dot{V}(\tau, \mu(\tau)) \leq-\varphi_{3}(\|\mu(\tau)\|),
\end{aligned}
$$

then (4) is asymptotically stable. 


\section{Control Law Design}

Define the tracking errors as follows:

$$
\left\{\begin{array}{l}
z_{1}=e_{1} \\
z_{2}=e_{2}-\alpha_{1}^{c} \\
z_{3}=e_{3}-\alpha_{2}^{c} \\
\vdots \\
z_{n}=e_{n}-\alpha_{n-1}^{c}
\end{array}\right.
$$
[31]:

The finite time command filter is designed as follows

$$
\left\{\begin{array}{l}
\dot{\varphi}_{i, 1}=v_{i, 1}, \\
v_{i, 1}=-r_{i, 1}\left|\varphi_{i, 1}-\alpha_{i}\right|^{1 / 2} \operatorname{sign}\left(\varphi_{i, 1}-\alpha_{i}\right)+\varphi_{i, 2}, \\
\dot{\varphi}_{i, 2}=-r_{i, 2} \operatorname{sign}\left(\varphi_{i, 2}-v_{i, 1}\right), \quad i=1, \ldots, n-1,
\end{array}\right.
$$

in which $\alpha_{i}$ is the input and $x_{i+1, c}(t)=\varphi_{i, 1}(t)$ and $\dot{x}_{i+1, c}(t)=$ $v_{i, 1}(t)$ are the outputs.

Remark 2 (see [32]). Note that parameters $r_{i, 1}$ and $r_{i, 2}$ should be large enough; $r_{i, 2}$ is selected first.

Construct the virtual signals $\alpha_{i}$ during the procedure of the finite time command-filtered backstepping control design as follows:

$$
\left\{\begin{array}{l}
\alpha_{1}=-k_{1} z_{1}-l_{1}\left(\widetilde{w}_{1}(t)\right) \\
\alpha_{2}=-k_{2} z_{2}-l_{2}\left(\widetilde{w}_{2}(t)\right)-z_{1}+\dot{\alpha}_{1}^{c} \\
\vdots \\
\alpha_{i}=-k_{i} z_{i}-l_{i}\left(\widetilde{w}_{i}(t)\right)-z_{i-1}+\dot{\alpha}_{i-1}^{c} \\
\alpha_{n}=-k_{n} z_{n}-l_{n}\left(\widetilde{w}_{n}(t)\right)-z_{n-1}+\dot{\alpha}_{n-1}^{c}
\end{array}\right.
$$

where $k_{i}$ are positive constants.

Proof. Step 1: Define the Lyapunov function as

$$
V_{1}=\frac{1}{2} z_{1}^{2}
$$

Taking the derivative of $V_{1}$ yields,

$$
\begin{aligned}
\dot{V}_{1} & =z_{1} \dot{z}_{1}=z_{1} \dot{e}_{1}, \\
& =z_{1}\left(e_{2}+l_{1}\left(\tilde{x}_{1}(t), \tilde{y}_{1}(t)\right)\right) \\
& =z_{1}\left(e_{2}+l_{1}\left(\widetilde{w}_{1}(t)\right)-\alpha_{1}+\alpha_{1}-\alpha_{1}^{c}+\alpha_{1}^{c}\right) \\
& =z_{1}\left(z_{2}-k_{1} z_{1}++\alpha_{1}^{c}-\alpha_{1}\right) \\
& =-k_{1} z_{1}^{2}+z_{1} z_{2}+z_{1}\left(\alpha_{1}^{c}-\alpha_{1}\right) .
\end{aligned}
$$

Step 2: Define the Lyapunov function as

$$
V_{2}=V_{1}+\frac{1}{2} z_{2}^{2}
$$

Taking the derivative of $V_{2}$ yields,

$$
\begin{aligned}
\dot{V}_{2}= & \dot{V}_{1}+z_{2} \dot{z}_{2}=\dot{V}_{1}+z_{2}\left(\dot{e}_{2}-\dot{\alpha}_{1}^{c}\right) \\
= & \dot{V}_{1}+z_{2}\left(e_{3}+l_{2}\left(\widetilde{w}_{2}(t)\right)-\dot{\alpha}_{1}^{c}\right) \\
= & \dot{V}_{1}+z_{2}\left(e_{3}+l_{2}\left(\widetilde{w}_{2}(t)\right)-\alpha_{2}+\alpha_{2}-\alpha_{2}^{c}+\alpha_{2}^{c}-\dot{\alpha}_{1}^{c}\right) \\
= & \dot{V}_{1}+z_{2}\left(z_{3}+\alpha_{2}^{c}-\alpha_{2}-k_{2} z_{2}-z_{1}\right) \\
= & -k_{1} z_{1}^{2}+z_{1} z_{2}+z_{1}\left(\alpha_{1}^{c}-\alpha_{1}\right)-k_{2} z_{2}^{2}+z_{2} z_{3} \\
& -z_{1} z_{2}+z_{2}\left(\alpha_{2}^{c}-\alpha_{2}\right) \\
= & -k_{1} z_{1}^{2}-k_{2} z_{2}^{2}+z_{2} z_{3}+z_{1}\left(\alpha_{1}^{c}-\alpha_{1}\right)+z_{2}\left(\alpha_{2}^{c}-\alpha_{2}\right)
\end{aligned}
$$

Step k: Define the Lyapunov function as

$$
V_{k}=V_{k-1}+\frac{1}{2} z_{k}^{2}
$$

Then, taking the derivative of $V_{k}$ yields,

$$
\begin{aligned}
\dot{V}_{k} & =\dot{V}_{k-1}+z_{k} \dot{z}_{k}=\dot{V}_{k-1}+z_{k}\left(\dot{e}_{k}-\dot{\alpha}_{k-1}^{c}\right), \\
& =\dot{V}_{k-1}+z_{k}\left(e_{k+1}+l_{k}\left(\widetilde{w}_{k}(t)\right)-\dot{\alpha}_{k-1}^{c}\right) \\
& =\dot{V}_{k-1}+z_{k}\left(e_{k+1}+l_{k}\left(\widetilde{w}_{k}(t)\right)-\alpha_{k}+\alpha_{k}-\alpha_{k}^{c}+\alpha_{k}^{c}-\dot{\alpha}_{k-1}^{c}\right) \\
& =\dot{V}_{k-1}+z_{k}\left(-k_{k} z_{k}+z_{k+1}-z_{k-1}+\alpha_{k}^{c}-\alpha_{k}\right) \\
& =\dot{V}_{k-1}-k_{k} z_{k}^{2}+z_{k} z_{k+1}-z_{k-1} z_{k}+z_{k}\left(\alpha_{k}^{c}-\alpha_{k}\right) \\
& =-\sum_{i=1}^{k} k_{i} z_{i}^{2}+z_{k} z_{k+1}+\sum_{i=1}^{k} z_{i}\left(\alpha_{i}^{c}-\alpha_{i}\right) .
\end{aligned}
$$

Step n: For the $n$th subsystem, define the Lyapunov function as

$$
V_{n}=V_{n-1}+\frac{1}{2} z_{n}^{2}
$$

Then, it follows that,

$$
\begin{aligned}
\dot{V}_{n} & =\dot{V}_{n-1}+z_{n} \dot{z}_{n}=\dot{V}_{n-1}+z_{n}\left(\dot{e}_{n}-\dot{\alpha}_{n-1}^{c}\right), \\
& =\dot{V}_{n-1}+z_{n}\left(l_{n}\left(\widetilde{w}_{n}(t)\right)+u-\dot{\alpha}_{n-1}^{c}\right) \\
& =\dot{V}_{n-1}-k_{n} z_{n}^{2}-z_{n-1} z_{n} \\
& =-\sum_{i=1}^{n} k_{i} z_{i}^{2}+\sum_{i=1}^{n-1} z_{i}\left(\alpha_{i}^{c}-\alpha_{i}\right) .
\end{aligned}
$$

Step $n+1$ : Finally,

$$
\forall \mu>0, \quad \exists t_{0},
$$

where $r_{i, 2}$ is large enough; we have

$$
t>t_{0}, \quad\left|\alpha_{i}^{c}-\alpha_{i}\right| \leq \mu,
$$

that means $\alpha_{i}^{c}-\alpha_{i}$ is bounded.

$$
\dot{V}_{n} \leq-\sum_{i=1}^{n} k_{i} z_{i}^{2}+\mu .
$$




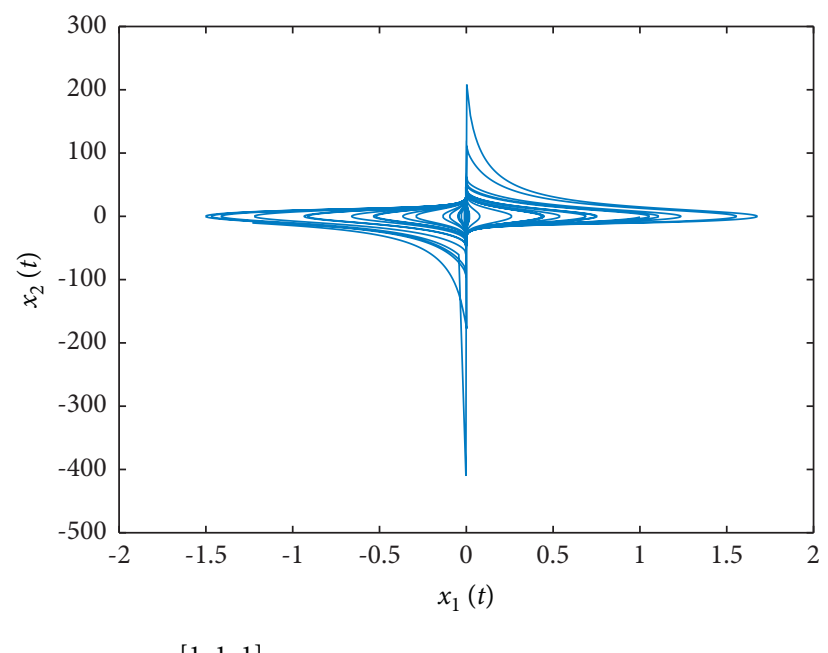

FIGURE 1: Chaotic phenomenon of integer-order gyro system (21).

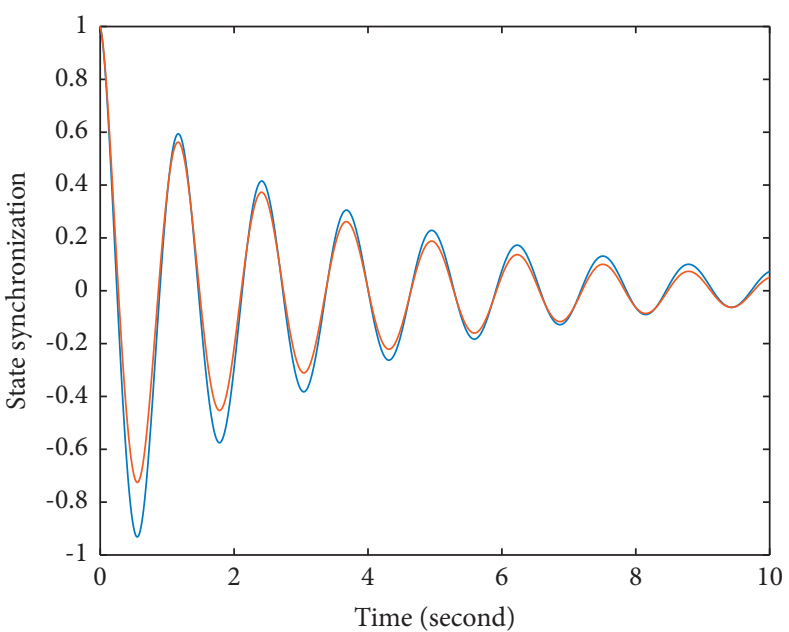

(a)

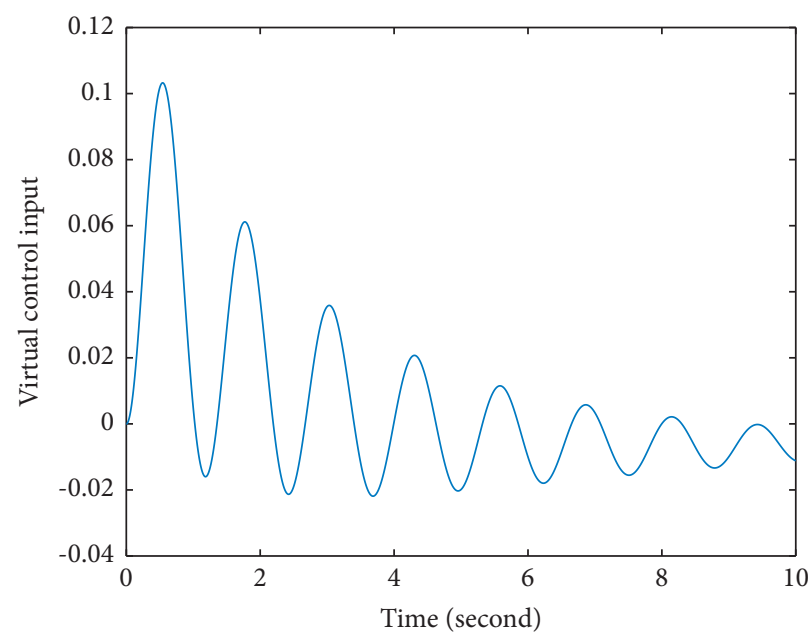

(b)

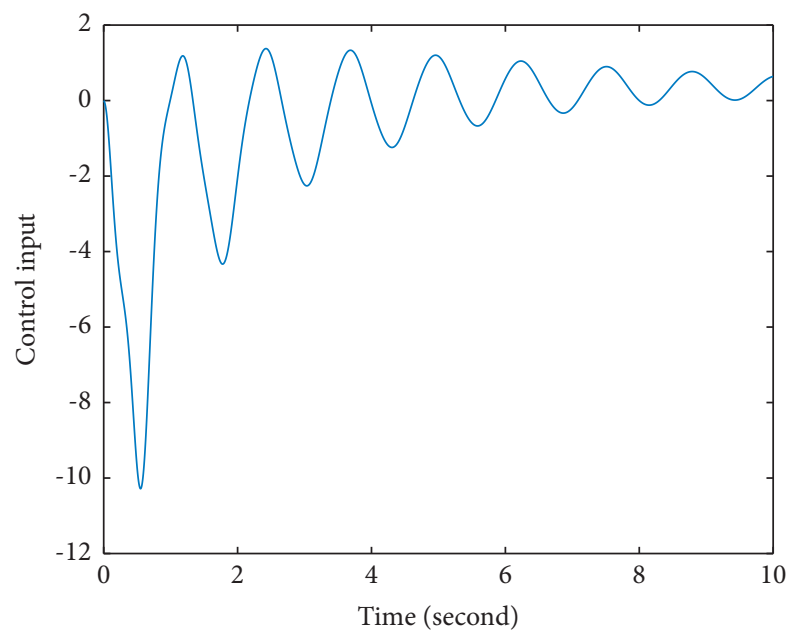

(c)

FIGURE 2: Simulation results: (a) state synchronization; (b) virtual control input; (c) control input. 
From (5), it satisfies the stability characteristic. Therefore, we can get the following theorem.

Theorem 1. Consider system (3). The finite time command filter and the virtual signals $\alpha_{i}$ are given in (8) and (9), respectively. If the choice of $\mu$ in (18) is large enough, then the system is asymptotically stable so that system (1) and system (2) are synchronized.

\section{Simulation Results}

A simulation example is given to declare the availability of the propound controller in this section. We selected the master and slave systems as follows [33, 34].

\subsection{Master System.}

$$
\left\{\begin{array}{l}
\dot{x}_{1}(\tau)=x_{2}(\tau) \\
\dot{x}_{2}(\tau)=-a^{2}\left(1-\cos x_{1}(\tau)\right)^{2} \sin ^{-3} x_{1}(\tau)-c_{1} x_{2}(\tau)-c_{2} x_{2}^{3}(\tau)+b \sin x_{1}(\tau)+c \sin \omega \tau \sin x_{1}(\tau) .
\end{array}\right.
$$

\subsection{Slave System.}

$$
\left\{\begin{array}{l}
\dot{y}_{1}(\tau)=y_{2}(\tau) \\
\dot{y}_{2}(\tau)=u(\tau)-a^{2}\left(1-\cos y_{1}(\tau)\right)^{2} \sin ^{-3} y_{1}(\tau)-c_{1} y_{2}(\tau)-c_{2} y_{2}^{3}(\tau)+b \sin y_{1}(\tau)+c \sin \omega \tau \sin y_{1}(\tau)
\end{array}\right.
$$

In system (21), we select the parameter as $a^{2}=100, b=$ $1, c_{1}=0.5, c_{2}=0.05, c=35.5, \omega=25$ and the initial condition $x(0)=[-1,1]^{T}$, which shows chaotic phenomenon in Figure 1.

In the simulation, the simulation results are shown in Figure 2.

\section{Conclusion}

In this paper, a command filter backstepping controller is designed, which gives full play to the advantages of backstepping control in dealing with nonlinear systems. Firstly, the system backstepping controller is designed according to Lyapunov stability theory, and then the constrained command filter is introduced to solve the control saturation problem existing in the application of traditional backstepping control engineering, and a controller is constructed so that the synchronization error can asymptotically approach zero in a short time. Simulation results show that the algorithm has better power tracking performance and dynamic response ability and is more suitable for engineering applications.

\section{Data Availability}

The data used to support the findings of this study are available from the author upon request.

\section{Conflicts of Interest}

The author declares no conflicts of interest.

\section{References}

[1] J. Zhang and J. Wang, "Numerical analysis for Navier-Stokes equations with time fractional derivatives," Applied Mathematics and Computation, vol. 336, pp. 481-489, 2018.
[2] G. Li, J. Cao, A. Alsaedi, and B. Ahmad, "Limit cycle oscillation in aeroelastic systems and its adaptive fractional-order fuzzy control," International journal of machine learning and cybernetics, vol. 9, no. 8, pp. 1297-1305, 2018.

[3] H. Liu, S. Li, J. Cao, G. Li, A. Alsaedi, and F. E. Alsaadi, “Adaptive fuzzy prescribed performance controller design for a class of uncertain fractional-order nonlinear systems with external disturbances," Neurocomputing, vol. 219, pp. 422-430, 2017.

[4] I. Pan and S. Das, "Fractional order fuzzy control of hybrid power system with renewable generation using chaotic pso," ISA Transactions, vol. 62, pp. 19-29, 2016.

[5] P. Wang, G. Wen, X. Yu, W. Yu, and W. Zhang, "Fully distributed consensus tracking of multiagent systems with a high-dimensional leader and directed communication topology," IEEE Transactions on Circuits and Systems II: Express Briefs, vol. 66, no. 8, pp. 1431-1435, 2019.

[6] Y. Pan and H. Yu, "Biomimetic hybrid feedback feedforward neural-network learning control," IEEE Transactions on Neural Networks and Learning Systems, vol. 28, no. 6, pp. 1481-1487, 2016.

[7] Y. Pan, C. Yang, L. Pan, and H. Yu, "Integral sliding mode control: performance, modification, and improvement," IEEE Transactions on Industrial Informatics, vol. 14, no. 7, pp. 3087-3096, 2017.

[8] H. Liu, S. Li, H. Wang, and Y. Sun, "Adaptive fuzzy control for a class of unknown fractional-order neural networks subject to input nonlinearities and dead-zones," Information Sciences, vol. 454-455, pp. 30-45, 2018.

[9] Y. Li, S. Tong, and T. Li, "Hybrid fuzzy adaptive output feedback control design for uncertain mimo nonlinear systems with time-varying delays and input saturation," IEEE Transactions on Fuzzy Systems, vol. 24, no. 4, pp. 841-853, 2015.

[10] Y.-J. Liu and S. Tong, "Barrier lyapunov functions for nussbaum gain adaptive control of full state constrained nonlinear systems," Automatica, vol. 76, pp. 143-152, 2017.

[11] A. Boulkroune, "A fuzzy adaptive control approach for nonlinear systems with unknown control gain sign," $\mathrm{Neu}$ rocomputing, vol. 179, pp. 318-325, 2016. 
[12] P. Wang, G. Wen, X. Yu, W. Yu, and T. Huang, "Synchronization of multi-layer networks: from node-to-node synchronization to complete synchronization," IEEE Transactions on Circuits and Systems I: Regular Papers, vol. 66, no. 3, pp. 1141-1152, 2019.

[13] G. Espinoza and E. Dz, Designing and Implementing an Autonomous Navigation System Based on Extended Kalman Filter in a Corobot Mobile, Springer International Publishing, Manhattan, NY, USA, 2015.

[14] M. K. Shukla and B. B. Sharma, "Backstepping based stabilization and synchronization of a class of fractional order chaotic systems," Chaos, Solitons \& Fractals, vol. 102, pp. 274-284, 2017.

[15] Y. Pan, H. Wang, X. Li, and H. Yu, "Adaptive commandfiltered backstepping control of robot arms with compliant actuators," IEEE Transactions on Control Systems Technology, vol. 26, no. 3, pp. 1149-1156, 2017.

[16] C. Kwan and F. L. Lewis, "Robust backstepping control of nonlinear systems using neural networks," IEEE Transactions on Systems, Man, and Cybernetics - Part A: Systems and Humans, vol. 30, no. 6, pp. 753-766, 2000.

[17] H. Li, L. Wang, H. Du, and A. Boulkroune, "Adaptive fuzzy backstepping tracking control for strict-feedback systems with input delay," IEEE Transactions on Fuzzy Systems, vol. 25, no. 3, pp. 642-652, 2016.

[18] F. Bribiesca-Argomedo and M. Krstic, "Backstepping-forwarding control and observation for hyperbolic pdes with fredholm integrals," IEEE Transactions on Automatic Control, vol. 60, no. 8, pp. 2145-2160, 2015.

[19] D. Swaroop, J. K. Hedrick, P. P. Yip, and J. C. Gerdes, "Dynamic surface control for a class of nonlinear systems," IEEE Transactions on Automatic Control, vol. 45, no. 10, pp. 1893-1899, 2000.

[20] T. P. Zhang and S. S. Ge, "Adaptive dynamic surface control of nonlinear systems with unknown dead zone in pure feedback form," Automatica, vol. 44, no. 7, pp. 1895-1903, 2008.

[21] J. A. Farrell, M. Polycarpou, M. Sharma, and W. Wenjie Dong, "Command filtered backstepping," IEEE Transactions on Automatic Control, vol. 54, no. 6, pp. 1391-1395, 2009.

[22] W. Wenjie Dong, J. A. Farrell, M. M. Polycarpou, V. Djapic, and M. Sharma, "Command filtered adaptive backstepping," IEEE Transactions on Control Systems Technology, vol. 20, no. 3, pp. 566-580, 2012.

[23] Y. Li and S. Tong, "Command-filtered-based fuzzy adaptive control design for mimo-switched nonstrict-feedback nonlinear systems," IEEE Transactions on Fuzzy Systems, vol. 25, no. 3, pp. 668-681, 2016.

[24] J. Yu, P. Shi, W. Dong, and H. Yu, "Observer and commandfilter-based adaptive fuzzy output feedback control of uncertain nonlinear systems," IEEE Transactions on Industrial Electronics, vol. 62, no. 9, pp. 5962-5970, 2015.

[25] H. Q. Wang, B. Chen, and C. Lin, "Approximation-based adaptive fuzzy control for a class of non-strict-feedback stochastic nonlinear systems," Science China(Information Sciences), vol. 3, p. 16, 2014.

[26] S. Sui, C. P. Chen, and S. Tong, "Neural network filtering control design for nontriangular structure switched nonlinear systems in finite time," IEEE Transactions on Neural Networks and Learning Systems, vol. 30, no. 7, pp. 2153-2162, 2018.

[27] Z. Wang, X. Jin, L. Pan, Y. Feng, and J. Cao, "Quasi-synchronization of delayed stochastic multiplex networks via impulsive pinning control," IEEE Transactions on Systems, Man, and Cybernetics: Systems, no. 1-9, 2021.
[28] Z. Wang, H. He, G.-P. Jiang, and J. Cao, "Quasi-synchronization in heterogeneous harmonic oscillators with continuous and sampled coupling," IEEE Transactions on Systems, Man, and Cybernetics: Systems, vol. 51, no. 2, pp. 1267-1277, 2021.

[29] S. P. Bhat and D. S. Bernstein, "Finite-time stability of continuous autonomous systems," SIAM Journal on Control and Optimization, vol. 38, no. 3, pp. 751-766, 2000.

[30] Y. Li, Y. Chen, and I. Podlubny, "Mittag-leffler stability of fractional order nonlinear dynamic systems," Automatica, vol. 45, no. 8, pp. 1965-1969, 2009.

[31] J. Yu, P. Shi, and L. Zhao, "Finite-time command filtered backstepping control for a class of nonlinear systems," Automatica, vol. 92, pp. 173-180, 2018.

[32] A. Levant, "Higher-order sliding modes, differentiation and output-feedback control," International Journal of Control, vol. 76, no. 9-10, pp. 924-941, 2003.

[33] M. P. Aghababa and H. P. Aghababa, "The rich dynamics of fractional-order gyros applying a fractional controller," Proceedings of the Institution of Mechanical Engineers - Part I: Journal of Systems \& Control Engineering, vol. 227, no. 7, pp. 588-601, 2013.

[34] S. Ha, H. Liu, S. Li, and A. Liu, "Backstepping-based adaptive fuzzy synchronization control for a class of fractional-order chaotic systems with input saturation," International Journal of Fuzzy Systems, vol. 21, no. 5, pp. 1571-1584, 2019. 